\title{
Tailored electrospun fibers from waste polystyrene for high oil adsorption
}

\author{
Tuğba Isık, Mustafa M. Demir * \\ Department of Materials Science and Engineering, Izmir Institute of Technology, 35430 Urla, Izmir, Turkey
}

\section{A R T I C L E I N F O}

\section{Article history:}

Received 25 August 2018

Received in revised form 7 November 2018

Accepted 7 November 2018

\section{Keywords:}

Expanded polystyrene

Oily wastewater

Remediation

Recycling

Waste-electrospinning

Waste-PS

\begin{abstract}
A B S T R A C T
Recent ship accidents that resulted catastrophic oil spills necessitate producing environmentally friendly, costeffective, and large-scale fabrication technology for oil- sorbent materials. Various material systems have been employed to fabricate sorbent materials; however, using fresh material components as adsorbent can lead to a secondary pollution. Therefore, recycling of plastics wastes for the fabrication of adsorbent material could be a wise approach to handle this environmental issue. In this study, foam-expanded polystyrene (f-PS), a commodity polymer used for insulation and packing materials, was electrospun from solution mixture of THF and DMF. Surface and interior porosity were achieved from individual fibers electrospun from a composition of DMF:THF (1:3) at 20 -wt\% of solid f-PS content. The resulting adsorbents exhibited a considerable hydrophobicity (WCA $\approx 120^{\circ}$ ) and oleophilicity $\left(\mathrm{CA} \approx 10^{\circ}\right.$ ), which can selectively adsorb both vegetable and engine oils from polluted waters. The porosity of the fibers has significant effect on the sorption capacity and separation efficiency up to $124 \mathrm{~g} / \mathrm{g}$ and $99 \%$, respectively. Thus, electrospun mats of the polystyrene wastes offer a promising adsorbent for the remediation of oily wastewaters.
\end{abstract}

(c) 2018 Published by Elsevier B.V.

\section{Introduction}

A remarkable increase in the amount of oily wastewater originating from various sources particularly sea accidents brings front serious environmental issues that threaten human life and ecological systems [1-5]. According to a report of National Academy of Sciences, nearly $343,200,000$ gal of oil were spilled into seas annually. [6] Conventional oil-water separation methods vary from gravity separation, [7] centrifugal settling, [8] air flotation, [9,10] and chemical demulsification [11]; however, they have inherent drawbacks such as low efficiency, need of large footprint, consumption of high energy, equipment corrosion, high equipment cost, and secondary pollution. Thus, there is an outgrowth environmental demand for the oily wastewater treatment systems, which are highly selective, efficient and effortless to apply. In recent years, filtration membranes have been developed for the remediation of oily wastewater. However, membrane-fouling problems that reduce the separation efficiency, and high cost limit the applicability of these materials. [12] Therefore, the fabrication of low-cost adsorbent materials showing special wettability to oil arises a significant attention. $[3,13,14]$ This feature determines the interaction between liquid and solid surface and wettability originating from the oleophilic and hydrophobic character of the sorbent surfaces. Oil removing type of adsorbents can selectively adsorb oil from oil/water mixtures and this

\footnotetext{
* Corresponding author.

E-mail address: mdemir@iyte.edu.tr (M.M. Demir).
}

property is ascribed to their superhydrophobic/superoleophilic nature. [12] This type of adsorbents can convert liquid state oily wastes into semisolid state and enables the ease removal of oil from the medium. $[15,16]$ Diverse methods have been proposed for the fabrication of this kind of novel materials by rational combination of surface composition and rough structure such as sol-gel [17], salt induced phase inversion [18], layer-by-layer assembly $[19,20]$, chemical or plasma etching [21,22], and electrochemical processes [23]. Among all the current techniques, electrospinning is one of the most versatile techniques used to fabricate hydrophobic surfaces. [24,25] It is a straightforward method for the fabrication of nonwoven micro-/nanoscale fibers and utilization of the electrospun mats as adsorbents in various media. [26-31] The structural advances of the mats stem from their enhanced surface area, promising mechanical integrity, and tunable surface features. [32-36] Since the nonwoven fibers present micrometer-sized interfibrillar spacing, the fibrous mats have surface roughness that trap air and could minimize the interfacial energy between solid sorbents and liquid oily water [25,37].

There are plenty of material systems that have been used for oil separation from aqueous systems such as inorganic adsorbents (silica aerogels, zeolites, clays), [12] organosilane surfaces, [38] poly(vinylidene fluoride), [39] polycarbonate monolith, [40] poly acrylonitrile, [41,42] polyurethane, [43] modified polyurethane. [44] Zhang et al. demonstrated the low oil adhesion property of successfully fabricated pindle-knotted structured graphene oxide - polyacrylonitrile composite fibers and graphene oxide modified polyacrylonitrile fibers by 
electrospinning. [41,42] Among them, polystyrene (PS) is known with its low cost, high chemical resistance as well as hydrophobicity. The hydrophobic property brings about the oleophilic feature for PS adsorbents. There are numerous routes for the fabrication of PS adsorbents in oil spill cleanup applications but many of the existing routes challenge for easy to use due to the multistep procedures. [45] For instance, Lee et al. fabricated electrospun PS nanofibers onto stainless steel mesh for the removal of oil spills and PS membranes exhibited high efficiency to remove many types of organic solvents or oils from water at large production scales. [46] Other studies also elucidate the utilization of PS fibers for oily wastewater remediation. [47,48]

Note that electrospinning of PS allows controlling both surface and internal porosity of an individual fiber along with the roughness of electrospun mat (assembly of the nonwoven individual fibers) [49]. During the electrospinning of polymer solutions, solvent evaporation occurs rapidly and thermodynamically driven events dominantly come into play. [50] Phase separation is a common strategy, particularly in humid environments, for the development of porosity inside and/or on the fibers surface. Vapor-induced phase separation (VIPS) and thermal-induced phase separation (TIPS) are the most pertinent phase separation mechanisms that cause to the development of porosity formation on PS fibers. [51-54] In the former, moisture plays a significant role due to the non-solvent/solvent interaction such that water acts as a non-solvent for PS. This process is induced by penetration of nonsolvent from vapor phase into the polymer solution and non-solvent (water) precipitates the polymer out of the solution by generating a solid matrix from polymer-rich phase. The solvent-rich regions leave behind porosity. In the latter, liquid-liquid disintegration has significant importance; a phase separation takes place in polymer rich and solvent rich regions. [55] Phase separation in TIPS mechanism occurs by lowering the surface temperature of fibers due to the rapid evaporation of solvent and the system passes through the binodal curve to enter the metastable region. The porous structure of amorphous polymers comes from the liquid-liquid phase separation following by gelation of the polymer. [56] Moreover, rapid evaporative cooling on the surface of fibers could lead to the formation of the breath figure on the surface that cools the moisture in air and condenses by following the growth of droplets. As the jet dries, the water droplets leave imprints on the surface in the form of pores. [57,58] It can be claimed that neither the phase separation nor the formation of breath figures alone fully explains the pore formation in electrospun PS fibers. Rather, the combination of all mechanisms may simultaneously play active role in the structural development of the electrospun fibers.

There are limited numbers of researches for the fabrication of PS fibers from waste Styrofoam (expanded PS, f-PS). [59-61] In connection with the recycling problem of f-PS wastes, which comes from the long recycling periods and being a widely used material in industry, there is a need for the recycling of EPS wastes and reusing these wastes as secondary products. Herein, we reported a simple and low-cost method for the fabrication of hydrophobic oil adsorbent fibers from waste PS sources by electrospinning in $N, N$ - Dimethylformamide (DMF)/Tetrahydrofuran (THF) mixture. The solvent composition governs the morphology of the fibers such that the fibers have surface texture and/or interior porosity. Considering the growing number of oil-spill accidents and variable oil compositions, the adsorbents were examined for multiple types of oil. The morphology is optimized for the highest oil adsorption capacity for both vegetable and engine oils.

\section{Experimental section}

\subsection{Materials}

Expanded polystyrene (f-PS) wastes were used without further purification. $\mathrm{N}, \mathrm{N}$ - Dimethylformamide (DMF, 99\%, Carlo Erba) and Tetrahydrofuran (THF, Sigma - Aldrich) were used to dissolve the polymers.

\subsection{Adsorbent fabrication by electrospinning}

For the molecular weight determination of PS samples, refractive indices of PS solutions (150, 100, 50, and 25 g. . $^{-1}$ ) were determined using a manual Abbe Refractometer and the refractive index increment (dn. $\mathrm{dc}^{-1}$ ) of each PS sample was calculated. Then, these records were entered dynamic light scattering (DLS) instrument (Malvern Nano ZS, Worcestershire, UK). The scattering Rayleigh ratio of DMF was taken as $9.82 \AA \sim 10^{-6}$ and the weight-averaged molecular weight of samples was calculated. This procedure was conducted for virgin polystyrene (vir-PS) and waste foam PS (f-PS). Molecular weight of vir-PS and f-PS were found to be 322 and $40 \mathrm{kDa}$, respectively. Precursor electrospinning solutions of both vir-PS and f-PS were prepared in various compositions of DMF:THF mixture with various solid contents of 10-15-20-25-30-wt\%. Viscosity measurements of all samples were conducted using a rotational viscometer (Thermo Scientific HAAKE Viscotester C, Massachusetts, USA) with a L1 spindle, where the rotational velocity of the spindle was $20 \mathrm{rpm}$ for 10 -wt\% solution, $10 \mathrm{rpm}$ for $15-w t \%$ solution, 5 rpm for 20 -wt\% solution, 2 rpm for 25 -wt\% solution and $1 \mathrm{rpm}$ for $30-\mathrm{wt} \%$ solution. Each solution was then transferred into a $10 \mathrm{~mL}$ plastic syringe using a stainless-steel needle (18 gauges); the needle was connected horizontally to a high voltage supply under $12 \mathrm{kV}$ (Gamma High Voltage Research Ormond Beach, FL, US). A micro-infusion pump (New Era NE300 Infusion Pump, Farmingdale, NY, USA) was used to fix the flow rate at $2.0 \mathrm{~mL} \mathrm{~h}^{-1}$ and the tip to collector distance was set at $12 \mathrm{~cm}$; therefore, the potential difference was $1.0 \mathrm{kV} \mathrm{cm}^{-1}$. Humidity and temperature were recorded as $52 \%$ and 24 ${ }^{\circ} \mathrm{C}$, respectively. Nonwoven electrospun fibers were collected on aluminum foil.

\subsection{Fiber characterization}

The morphology of fibers was examined using a Scanning Electron Microscope (SEM, Carl Zeiss 300VP, Germany) after sputtering by gold using ion sputter. The average size of the fibers and pores were estimated by statistically from SEM micrographs using Image software. Contact angle measurements were achieved with Contact Angle Meter (Attension Lite, Biolin Scientific, Stockholm, Sweden) for both water and oil contact angles. The surface area of the electrospun mats was measured using the Brunauer-Emmett-Teller (BET) gas adsorption method (Micromeritics Gemini V, GA, USA). Surface chemistry of fibers was studied using an X-ray photoelectron spectrometer (XPS, Thermo Scientific K-Alpha Surface Analysis, Massachusetts, USA) and thermal degradation profile was determined by Perkin-Elmer Diamond TG/DTA.

\subsection{Oil adsorption capacity and oil separation efficiency of fibers}

For the comparison of the oil adsorption capacity of electrospun fibers, the maximum oil adsorption capacities of each type of fibers were studied. A sample of $25 \mathrm{mg}$ of each fiber mats was immersed in $10 \mathrm{~mL}$ of vegetable oil (which is stained with red oil paint) or engine oil. Then, the adsorbed oil amount was determined by measuring the difference between initial and final weight of the fibers with an analytical balance. All experiments were repeated 3 times to control the reproducibility. The results were given as adsorbed oil amount $(\mathrm{g})$ per gram fiber with their standard deviations. The required contact time for the maximum oil adsorption was determined $60 \mathrm{~s}$, which is the optimum time for the highest oil adsorption. (Fig. S1a) For each f-PS fiber, the oil adsorption capacity increases with adsorption time until the fibers reach saturation at $60 \mathrm{~s}$. Plots of $\ln \left(q_{e}-q_{t}\right)$ versus time were described in Fig. S2 and adsorption constant $(\mathrm{K})$ in Table S1 were obtained from the slope of pseudo order plots. The $\mathrm{K}$ value decreased with raising the polymer solution concentration from 10 to 20 -wt\% due to the higher oil adsorption capacity of f-PS-10 fibers. The fibers were taken in another vessel before measuring their weights and the excess oil on the fibers, which is not adsorbed by the fibrous material, was drained out. The 
required drain out time of fibers before performing gravimetric analysis was determined as $30 \mathrm{~s}$ for the reason of comparison with literature (Fig. S1b). The removal efficiency of oil from the aqueous mixtures was examined by preparing different concentrations of oil polluted water mixtures 2,5 , and $10 \%(\mathrm{v} / \mathrm{v})$ in $20 \mathrm{~mL}$ water by adding vegetable or engine oil droplets. An amount of $25 \mathrm{mg}$ of fiber was immersed in oily water and mixed for $60 \mathrm{~s}$. Then, the difference between initial and final weight of fibers was measured with an analytical balance. The results are reported as percentage oil separation efficiency with their standard deviations. The maximum oil adsorption capacity and oil water separation efficiency were examined for pristine foam and a commercial adsorbent, which was supplied by Municipality of İzmir, for the comparison of as-prepared fibers with the existing products.

\section{Results and discussion}

\subsection{Fabrication of PS adsorbents with tailored morphology}

Waste-EPS (f-PS) was dissolved in DMF and/or THF at various concentrations and subjected to electrospinning at $1.0 \mathrm{kV} \mathrm{cm}^{-1}$. Along with the neat solvents (DMF or THF), their binary mixtures (DMF:THF $=3: 1,1: 1$, and $1: 3$, by volume) were employed. Table 1 shows physical properties of all f-PS solutions and their corresponding fibers obtained by electrospinning of these solutions. Various morphologies of individual fibers (porous polymer cups, wrinkled beads-on-string, smooth fibers, wrinkled fibers and porous ribbon-shaped fibers) were achieved. Fig. 1 presents SEM micrographs of the f-PS fibers fabricated from the neat and binary solvent systems. The first column of the SEM images given in Fig. 1 shows the electrospun fibers obtained from neat DMF, $(D M F: T H F=4: 0)$ at different f-PS concentrations. The fibers favor 0.4 $\mu \mathrm{m}$ AFD with a smooth surface at 10 -wt\%. When the concentration increases to $30-w t \%$, AFD systematically increases up to $5.0 \pm 0.6 \mu \mathrm{m}$. It is well established that increasing the concentration, i.e. viscosity of the solution, increases the AFD. [62,63] At high concentration, the entanglements of the chains enhance the formation of stable jet with higher mass transfer of the electrospinning solution.

The first row of the SEM micrographs presented in Fig. 1 shows the fibers obtained from various compositions of the co-solvents DMF and
THF. Neat DMF provides straight fibers with uniform AFD without beads. Since DMF has high electrical conductivity, the electrospun jet is exposed to high electrical force and bead-free uniform fibers were obtained. On the other hand, binary solvent systems promote the formation of porosity through the fiber structure due the difference between evaporation rates of THF and DMF. The vapor pressures of solvents are $\sim 200 \mathrm{hPa}$ and $\sim 3.5 \mathrm{hPa}$, respectively. The addition of small portion THF (DMF:THF = 3:1) into the solvent system does not have remarkable effect on the surface texture such that electrospun fibers have still smooth surface as observed in fibers obtained from neat DMF. The equivolume mixture of DMF and THF (DMF:THF $=1: 1)$ results in the formation of wrinkled surface texture especially at high PS concentrations. Since the presence of THF in solvent system increases the vapor pressure and accelerates the solvent evaporation, the occurrence of surface texture occurs most probably due to the buckling of cylindrical polymer shell under compressive radial or a lateral contraction from the axial tensile stresses. [64] The rise in the amount of high vapor pressure solvent, THF, increases the solidification and evaporation rate of solvent system. [53] The fibers fabricated from 1:3 solvent (DMF:THF $=1: 3$ ) have large elongated pores on their surface. The development of this structure may arise from the rapid removal of solvent from the core of jet and its continuous stretching. The highly volatile solvent can rapidly form glassy skin and surface pores probably due to the phase separation and fast evaporation rate. Meanwhile, low volatile solvent still remains in the jet that makes the polymer core wet and stretchable. [65-67]

The neat THF system (DMF:THF $=0: 4$ ) causes the formation of a ribbon-like morphology with densely packed nanoscale surface pores (nearly $90 \mathrm{~nm}$ ) uniformly distributed over the beads and fibers. Although the most electrospun fibers are in cylindrical morphology, highly volatile solvents such as THF show ribbon-like morphology due to the rapid drying following by collapse of jet and this morphology results in the large AFD varying between 3.5 and $16 \mu \mathrm{m}$. [65] THF leads to the fabrication of porous polymer cups with $\sim 20 \mu \mathrm{m}$ size at low f-PS concentrations (10 to 20-wt\%). Bead and/or bead-on-string are observed when the polymer solution is fairly diluted because of the both insufficient viscosity and electrical conductivity of THF. The formation of pores may attribute to the both mechanisms of phase separations

Table 1

The characteristics of PS solutions and the resulting electrospun f-PS fibers.

\begin{tabular}{|c|c|c|c|c|c|c|}
\hline f-PS-wt\% & DMF & THF & Viscosity $(\mathrm{mPa} \cdot \mathrm{s})$ & Fiber morphology & Properties & Fiber diameter $(\mu \mathrm{m})$ \\
\hline \multirow[t]{5}{*}{10} & 4 & 0 & 24 & fiber & thin fiber & $0.4 \pm 0.1$ \\
\hline & 3 & 1 & 52 & fiber & thin fibers & $0.5 \pm 0.1$ \\
\hline & 1 & 1 & 44 & fiber & medium thickness fibers & $2.0 \pm 0.6$ \\
\hline & 1 & 3 & 34 & bead on string & medium fibers with macropores & $1.5 \pm 0.1$ \\
\hline & 0 & 4 & 28 & bead & porous polymer cups & - \\
\hline \multirow[t]{5}{*}{15} & 4 & 0 & 95 & fiber & medium thickness fibers & $1.0 \pm 0.2$ \\
\hline & 3 & 1 & 171 & fiber & medium thickness fibers & $1.9 \pm 0.6$ \\
\hline & 1 & 1 & 130 & fiber & medium thickness fibers with grooves & $2.5 \pm 0.4$ \\
\hline & 1 & 3 & 140 & bead on string & medium thickness fibers with grooves and macro-pores & $2.0 \pm 0.3$ \\
\hline & 0 & 4 & 88 & bead & porous polymer cups & - \\
\hline \multirow[t]{5}{*}{20} & 4 & 0 & 580 & fiber & medium thickness fibers & $1.8 \pm 0.3$ \\
\hline & 3 & 1 & 681 & fiber & thick fibers/interior porosity & $4.0 \pm 0.4$ \\
\hline & 1 & 1 & 554 & fiber & thick fibers/interior porosity & $3.5 \pm 0.6$ \\
\hline & 1 & 3 & 311 & fiber & thick fibers with grooves and macro-pores & $2.2 \pm 0.7$ \\
\hline & 0 & 4 & 279 & bead on string & thick/belt shape fibers with nanopores & $3.5 \pm 0.5$ \\
\hline \multirow[t]{5}{*}{25} & 4 & 0 & 1400 & fiber & thick fibers & $4.0 \pm 0.7$ \\
\hline & 3 & 1 & 2630 & fiber & thick fibers & $4.0 \pm 0.8$ \\
\hline & 1 & 1 & 1862 & fiber & thick fibers with grooves & $3.0 \pm 0.9$ \\
\hline & 1 & 3 & 756 & fiber & thick fibers with macro-pores & $3.5 \pm 0.9$ \\
\hline & 0 & 4 & 507 & fiber & thick/belt shape fibers with nanopores & $14.0 \pm 2.6$ \\
\hline \multirow[t]{5}{*}{30} & 4 & 0 & 2319 & fiber & thick fibers & $5.0 \pm 0.6$ \\
\hline & 3 & 1 & 5867 & fiber & thick fibers & $8.0 \pm 0.7$ \\
\hline & 1 & 1 & 2517 & fiber & thick fibers with grooves & $5.0 \pm 0.9$ \\
\hline & 1 & 3 & 2750 & fiber & thick fibers with grooves and macro-pores & $5.0 \pm 0.9$ \\
\hline & 0 & 4 & 2120 & fiber & thick/belt shape fibers with nanopores & $16.0 \pm 3.5$ \\
\hline
\end{tabular}




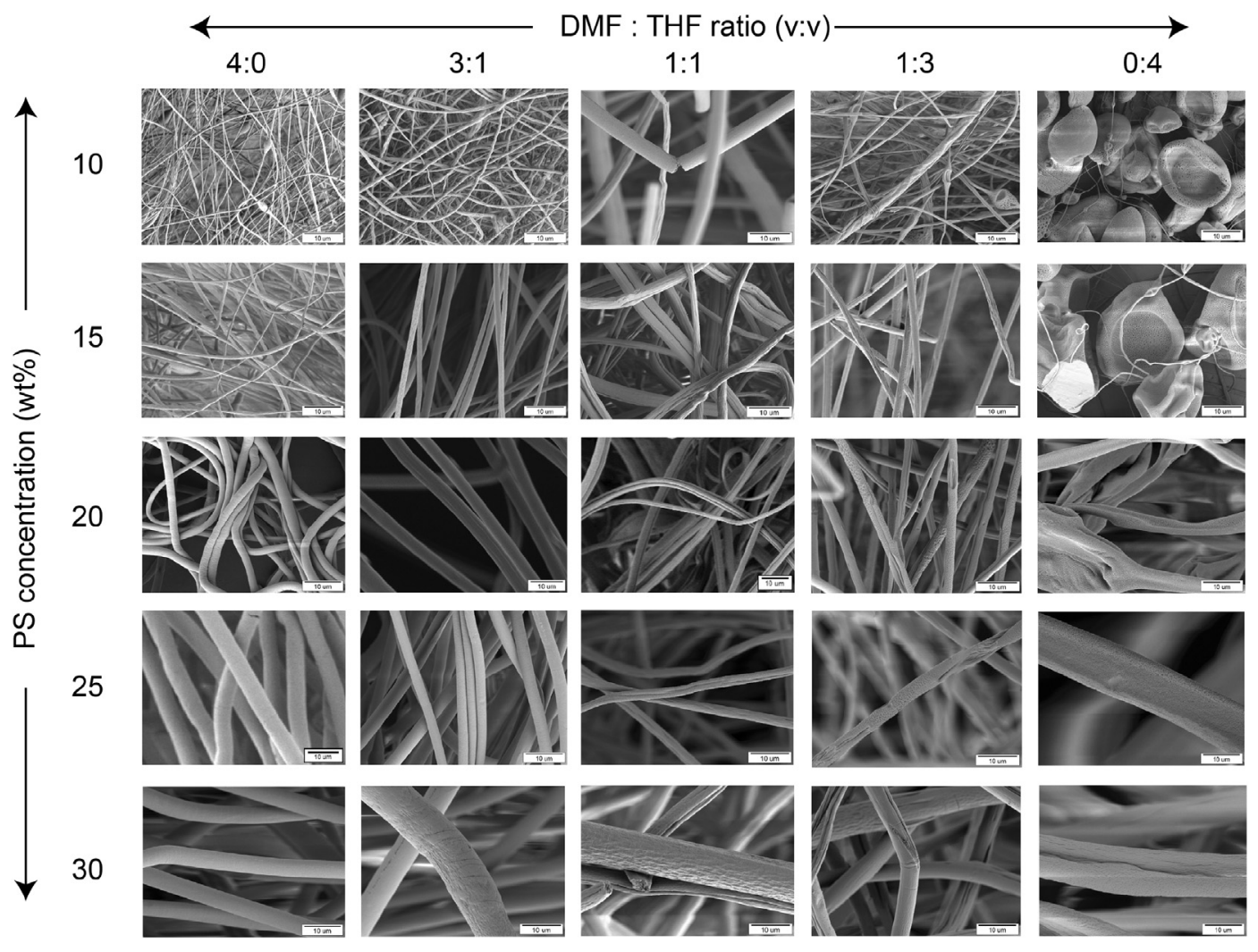

Fig. 1. SEM micrographs of the f-PS fibers with respect to various solvent compositions and f-PS solution concentrations (Scale bar is $10 \mu \mathrm{m}$ ).

(VIPS and TIPS) along with breath figure formation. Previously, Lu et al. proposed that VIPS plays a pivotal role in the formation of this structure that internal and external porous PS yarns were obtained. [57]

Fiber diameter is a function of solution properties so that AFD is examined as a function co-solvents composition. [25,27,31] A rise in the AFD was observed at all f-PS concentrations when the portion of THF increases in DMF. AFD is $1.0 \pm 0.2 \mu \mathrm{m}$ when neat DMF is used for spinning process of $15-w t \% \mathrm{f}-\mathrm{PS}$ solution. The addition of THF increases the diameter $1.9 \pm 0.6 \mu \mathrm{m}$ (DMF:THF $=3: 1$ ). The reason of increasing AFD could be the increase in viscosity upon incorporation of THF. DMF $(0.79 \mathrm{cP}$ at $\left.25^{\circ} \mathrm{C}\right)$ itself has higher viscosity than $\operatorname{THF}\left(0.46 \mathrm{cP}\right.$ at $\left.25^{\circ} \mathrm{C}\right)$ [68]. The viscosity of the electrospinning solution increases from 95 to $171 \mathrm{mPa} \cdot \mathrm{s}$ upon incorporation of THF for one portion. (Table 1) It is well established that viscosity is the dominant parameter in diameter of electrospun fibers. The mixture of co-solvents shows higher viscosity than the one of the corresponding polymer solution prepared by neat solvents either DMF or THF most probably due to the strong attraction of the both solvent molecules. The further increase of the amount of THF in DMF reduces the viscosity of the mixture at all f-PS concentrations. Thus, low viscosity of electrospinning solution favors the formation of thinner fibers with beads particularly observed at 10 and 15 -wt $\%$ f-PS concentrations.

Conductivity is another important parameter for electrospinning. Since the process takes place under high electrical field, the stretching of the spinning jet rigorously occurs for conductive solvents. [69] Dielectric constants of DMF and THF are 38.25 and 7.52, respectively. [68] Because of the lower electrical conductivity of THF, the addition of this cosolvent in the mixture inhibits the stretching of jet and results in larger AFD. As an example, a significant increase was observed from 1.8 to 4.0 $\mu \mathrm{m}$ when 20 -wt\% of f-PS electrospun from the solvents of $4: 0$ and 3:1 (DMF:THF), respectively. (Table 1).

Based on the overall data presented in Fig. 1, a map of various fiber surface textures as a function of the solvent composition and polymer solution concentration can be achieved. Fig. 2 shows mapping, in other words phase diagram of f-PS fiber morphology with respect to both parameters. The boundaries were constructed by estimation using the SEM micrographs. Polymer cups with surface porosity were observed from the 10 and 15 -wt\% f-PS concentrations in neat THF. On the other hand, the f-PS fibers fabricated from low portion of DMF (THF rich systems) at the same concentrations demonstrate wrinkled beads-on-string morphology. In addition to the elliptical-like beads, collapsed ones were also obtained depending on the vapor pressure of the solvent mixture. At low PS concentrations and neat THF solutions, collapsed beads were observed in Fig. 2. While DMF as low vapor pressure solvent systems form elliptical bead morphology, high vapor pressure solvent system shows collapsed beads. This variation can be attributed to the formation of instable fluid jet at low polymer concentration.

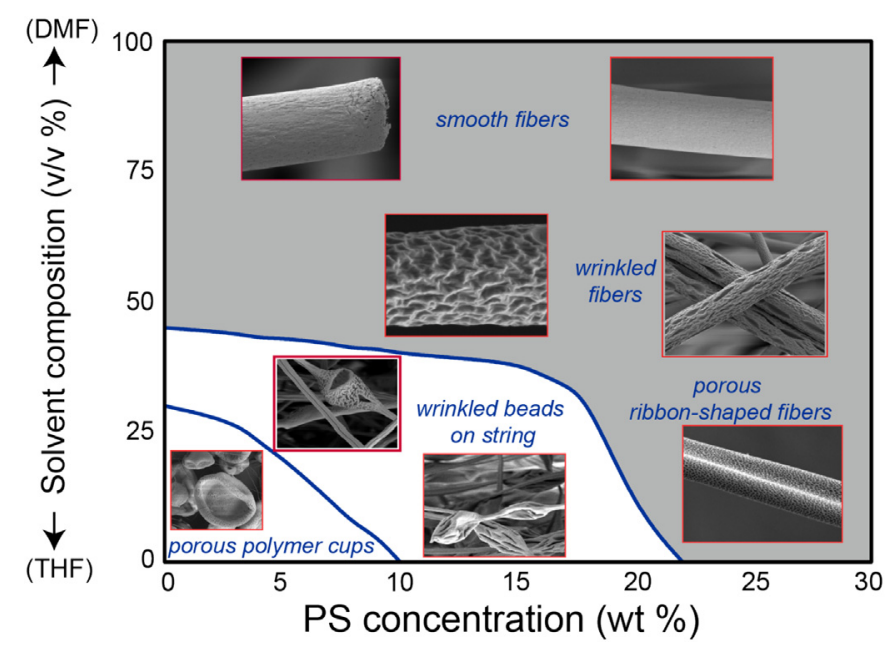

Fig. 2. Dependence of surface texture on DMF:THF composition versus f-PS solution concentration (solid lines were estimated by using the SEM micrographs in Fig. 1). 

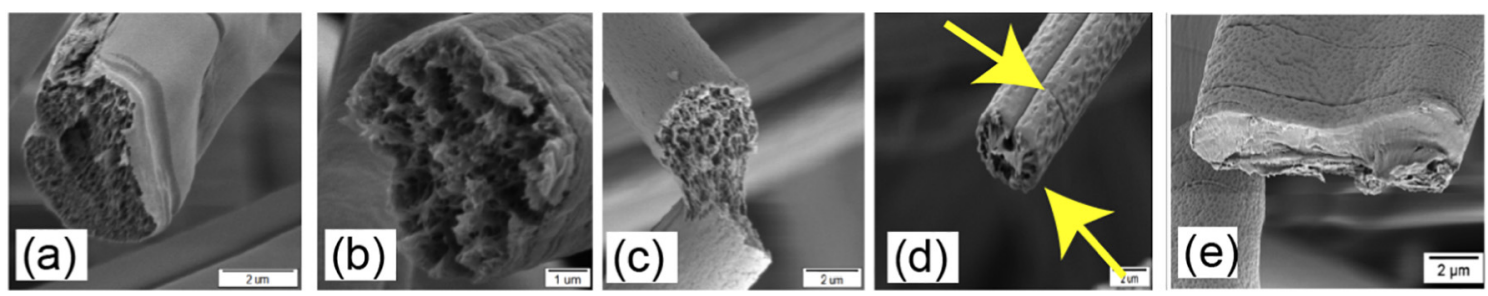

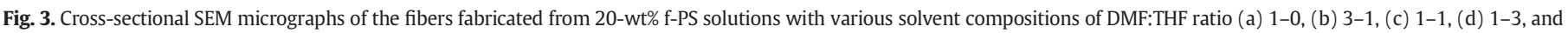
(e) $0-1$.

$[53,70]$ The grey region of the scheme illustrates the fibrous region that the fibers may have various morphological characteristics. From top to down, in other words from high to low DMF ratio, smooth fibers give their place to wrinkled and porous fibers probably due to the beforementioned phase separation mechanisms.

Fig. 3 shows the thin sheaths and porous cores of 20 -wt\% f-PS fibers electrospun from the various solvent compositions. The fibers fabricated from neat DMF or 3:1 solvent composition (DMF:THF) consist of interior porosity and fibrils aligned along the fiber axis most probably due to the longitudinal strain rate of jet during the electrospinning. [53] Water molecules are inevitably present in the solution system. One source could be the organic solvents because they are hygroscopic, i.e. absorb certain amount of water molecules. Another source could be humidity from the air, which is nearly $52 \%$ in the spinning environment. These water molecules play active role for the generation of interior porosity. DMF is trapped inside the electrospinning jet. Cooling of the jet upon solvent evaporation forms a glassy skin around the fibers such that the solvent molecules are unable to escape from the electrospinning jet. Water and DMF undergo a liquid-liquid phase separation where DMF is solvent; however, water is non-solvent. The removal of the water molecules causes the formation of the pores.

The increase in THF portion disappears the presence of interior porosity (Fig. 3d and e). However, the addition of THF into the electrospinning solution causes the formation of surface texture (porosity). The fibers fabricated from neat THF solvent do not have interior porosity. Since THF has a much higher vapor pressure than water, the jet - air interface was always saturated by THF even under high humidity, which effectively hindered the formation of a sheath as well as internal pores by preventing the water vapor from penetrating into the jet and inducing phase separation. Consistent with the literature, low vapor pressure of DMF plays a significant role in the generation of internal porosity, while THF plays a critical role on the surface texture of PS fibers with pores by discouraging the formation of interior pores.

There is a competitive penetration and diffusion of the three molecules (DMF, THF, and $\mathrm{H}_{2} \mathrm{O}$ ) and vapor has some primary effects, which are formation of thin but strong sheath due to the interface precipitation (VIPS and TIPS), internal phase separation due to the penetration of

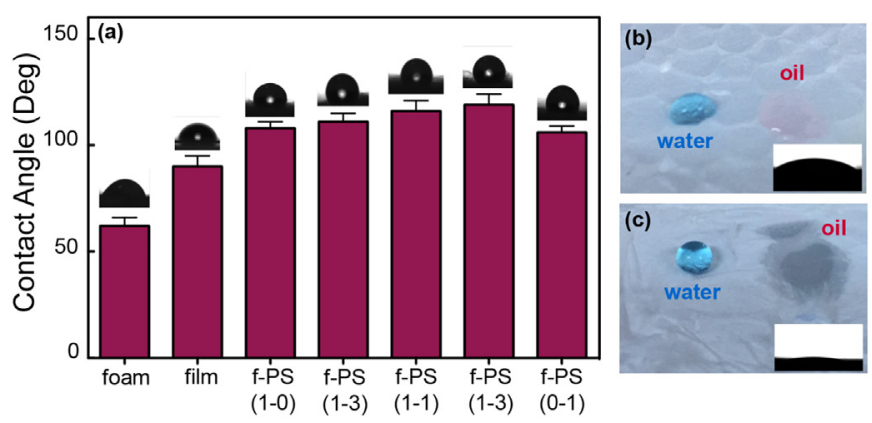

Fig. 4. (a) Water contact angle of the f-PS adsorbents electrospun from different solvent combinations with 20 -wt\% f-PS solution. Photographic images of water and oil droplets on (b) pristine foam and (c) f-PS fiber. water vapor (VIPS), and gathering of condensed water on the surface due to the evaporative cooling (breath figure formation). [57,58]

Electrospinning is a non-equilibrium process because the structure formation occurs in a very short period of time, on the order of $0.1 \mathrm{~s}$. [71] The stage at which the phenomenon of phase separation occurs is a true question. Considering high elongation rate and high speed of electrospun jet $(\approx 40 \mathrm{~m} / \mathrm{s}$ ), the penetration of water vapor is hard under these circumstances so that VIPS and/or TIPS should occur before the transportation of jet trough the collector presumably on solution droplet from which electrospinning is ejected. The rapid evaporation of solvent from the jet consumes the heat of vaporization by cooling the surface and leads the formation of thermodynamically unstable jets. The separation of phases as well as the breath figure formation may take place at this stage, where Taylor cone forms on the surface of a stationary solution droplet. [72,73]

\subsection{Water and oil contact angle of $f$-PS adsorbents}

For the investigation of hydrophobic/hydrophilic character of the fabricated fibrous materials, water contact angle (WCA) of the f-PS fiber mats fabricated from the various solvent compositions at $20-w t \%$ polymer concentration was measured. Fig. 4a demonstrates the dependence of WCA of the fibers with respect to the DMF:THF ratio along with foam itself and cast film of the pristine foam. The pristine foam presents WCA of $60^{\circ}$. The f-PS solution was cast on a glass slide and its WCA was also measured for comparison. WCA of the cast film is nearly 90, i.e. lies between the pristine foam and the fibrous electrospun mats. Because all the mats independent of the solvent compositions depict WCA $>100^{\circ}$ indicating that the fiber mats are hydrophobic. The hydrophobicity of the f-PS fiber mats may predominantly originate from the rough surface of the electrospun fibers. According to the Cassie-Baxter model, this roughness creates cavities on the surface and allows the trapping of air between surface and water droplet resulting in the inhibition of wetting the surface $[25,74,75]$. The fibers fabricated from 1:3 DMF to THF ratio demonstrated the highest WCA among all mats because more

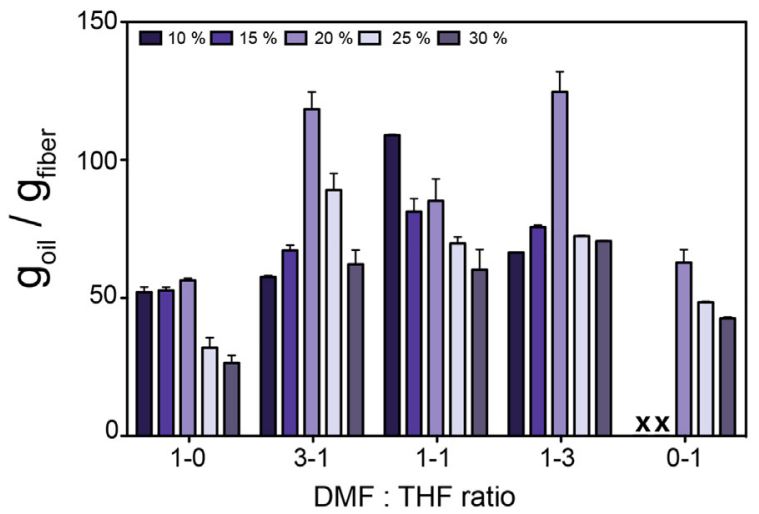

Fig. 5. Vegetable oil adsorption capacity of f-PS electrospun fibers with respect to solvent composition and initial PS concentration. The letters ' $x x$ ' refer to the absence of data points in the dataset probably due to the polymer cup formation and beads on string instead of fibers. 


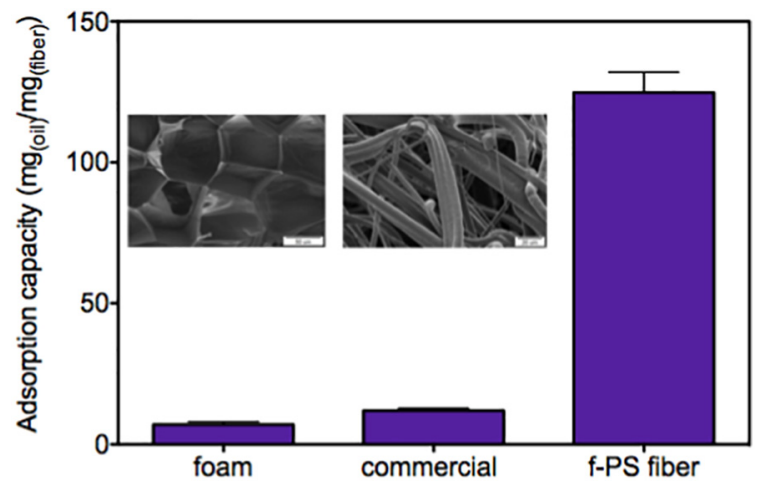

Fig. 6. Comparison of the vegetable oil adsorption capacity of pristine foam, commercial adsorbent and f-PS fibers fabricated from 1:3 (DMF:THF) solvent system showing the best sample consisting of both interior porosity and surface texture. Insets show the SEM micrographs of pristine foam and commercial adsorbent.

rough surfaces with cavities and surface texture show more hydrophobic behavior most probably due to the increment in the trapped air in cavities [76]. On the other hand, smooth fibers with neat DMF solvent not surprisingly show the lowest WCA among the all-fibrous mats.

The neat THF system resulted in lower WCA than the other fibers due to the ribbon-like cross section of the resulting fibers, which have fewer cavities than the circular ones. In conjunction with the hydrophobicity, the fibers also show oleophilicity. The photographs in Fig. 4b and c) show the wettability of water and oil droplets on the pristine foam and electrospun f-PS fiber mats, respectively. It is obviously seen that red stained vegetable oil quickly wets the surface of the f-PS fibers while the same amount of oil droplet stays on the pristine foam without being adsorbed. In addition, blue stained water droplet stays on the f-PS fiber surface in nearly round shape whilst the blue droplet spreads on the surface of the pristine foam. These results suggest that the f-PS fibers could be served as promising oil adsorbent. For further understanding of f-PS fibers' hydrophobic nature, their WCA hysteresis with respect to time has been observed in $60 \mathrm{~s}$.(Fig. S3) It is observed that the water droplets were not penetrated through the f-PS surfaces and remain nearly as it is during this time period.

\subsection{Oil adsorption capacity}

Fig. 5 demonstrates the oil adsorption capacity of the f-PS fibers with various surface textures. Whilst neat THF results in the polymer cups or wrinkled beads on string at low PS concentrations, the oil adsorption capacity of these samples could not be measured because the fiber mats did not have enough mechanical integrity. The oil adsorption capacity of f-PS fibers fabricated from 10-wt\% of PS concentration in equivolume mixture of DMF and THF solvent system is around $109 \mathrm{~g} / \mathrm{g}$; and the capacity decreases to $60 \mathrm{~g} / \mathrm{g}$ for the fibers obtained from 30-wt\% $\mathrm{f}$-PS concentration probably due to having less specific surface of thicker fibers. The thicker the fiber, the lower the surface area is.

When neat THF or DMF solvents were used for the fabrication of f-PS fibers obtained from 20-wt\% solutions, oil adsorption capacities lower than $60 \mathrm{~g}_{\mathrm{oil}} / \mathrm{g}_{\text {fiber }}$ were observed. The fibers in both cases have enclosed cross-section and smooth surface texture (Fig. 3a and e). On the other hand, f-PS fibers fabricated from binary solvent systems result in better oil adsorption capacity than neat DMF or THF ones. For instance, oil adsorption capacity of the f-PS fibers fabricated from 3:1 DMF:THF solvent composition reached up to $118 \mathrm{~g} / \mathrm{g}$. The reason could be the presence of interior porosity although these fibers have nearly smooth surface as in neat DMF solvent (Fig. 3b). The beads-on-string morphology of the fibers obtained from solvent composition of 1:3 DMF:THF does not have a significant effect on oil adsorption capacity. However, at 20-wt $\%$ PS concentration, the capacity reaches to its highest value although the fibers have thicker AFD. Normally the fibers are needed to be thin to have large surface area. However, the fibers should have enough mechanical integrity to resistance under continuous flow of liquids. The combination of both interior porosity and surface texture may enhance the permeability by contributing to a high oil adsorption. (Fig. 3d) The highest capacity was recorded for this sample as $124 \mathrm{~g} / \mathrm{g}$ indicating that $1 \mathrm{~g}$ of adsorbent could adsorb 124 times its own weight. Physical trapping of oil on the fiber surface and filling the voids between the individual fibers reduce the surface free energy of the fiber mats. The
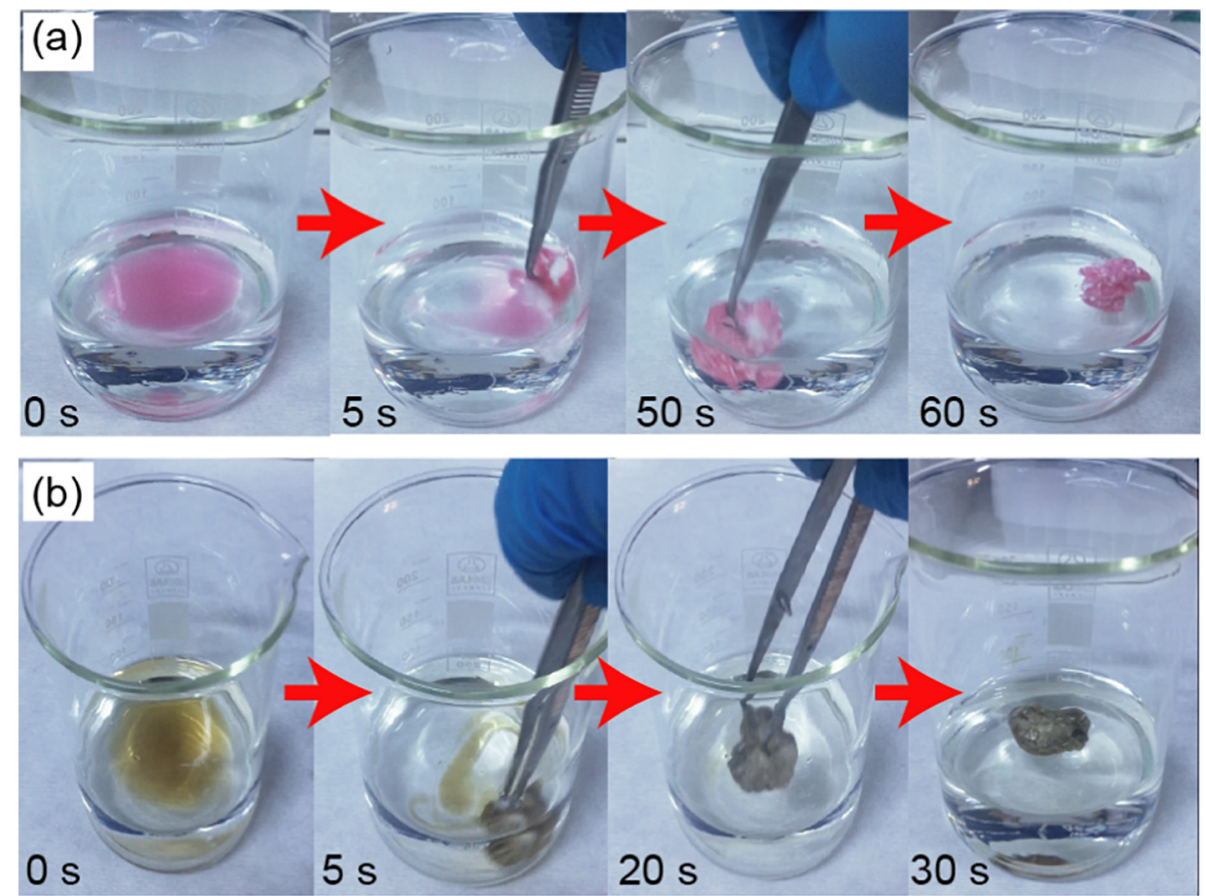

Fig. 7. The adsorption processes of (a) vegetable oil and (b) engine oil using the as-prepared f-PS adsorbent with $5 \%$ oil-polluted water 


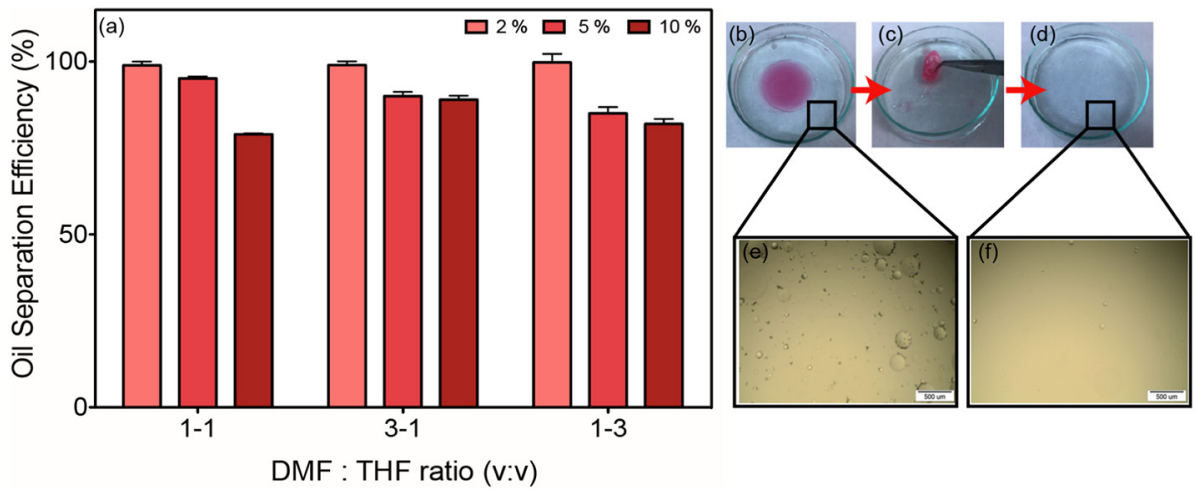

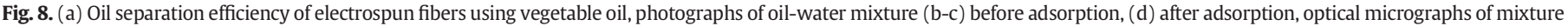
(e) before adsorption, and (f) after adsorption of $5 \%$ oil-polluted water.

voids between individual fibers and porous structure play a significant role on oil adsorption. $[48,77,78]$ The pores absolutely increase the specific surface area, which can be helpful for the adhesion and adsorption of oil on the fiber surface. The oleophilicity of f-PS fibers may play a pivotal role for the oil adsorption because the oleophilic nature of adsorbent serves a minimum energy barrier for oil to spread on the surface and finally diffuse into the fibrous adsorbent. $[45,79]$ Thus, the fibers prepared from DMF:THF mixture $(1,3)$ at 20 -wt\% PS have both interior porosity and surface texture. They are promising candidates for the fabrication of oil adsorbents, thanks to electrospinning.

The results were compared with the oil adsorption capacity of pristine foam and commercial adsorbent (Fig. 6). The pristine foam and commercial adsorbent show much lower oil adsorption capacity $(<15$ $\mathrm{mg}_{\text {oil }} / \mathrm{mg}_{\text {fiber }}$ ) than the fibrous f-PS adsorbents prepared in this work due to their low surface area and non-porous structure.

\subsection{Oil - Water separation efficiency}

Synthetic oily wastewater mixtures were prepared by changing the oil concentration in water from 2 to $10 \%(w / w)$ and oil separation efficiency of f-PS fibers were studied. Fig. 7 shows the process to capture of oil from water successfully by using the f-PS fibers. When a piece of the f-PS adsorbent brought into contact with oil-polluted mixture, the fiber floated on the water surface and adsorbed both red colored vegetable or engine oil selectively within a few seconds (Movie S1). After treatment with the f-PS adsorbent, the previously polluted water turned transparent and clean. Moreover, the fibrous adsorbents exhibited buoyancy that they were able to stay on the surface even after oil adsorption. Hereby, f-PS adsorbent stays compact after capturing the oil from water by conserving their mechanical integrity. This property of the f-PS mats is also important for the removal of adsorbents after oilspill cleanup.

Fig. 8a shows the comparison of oil separation efficiency of f-PS fibers fabricated from 20 -wt\% concentration from binary solvent systems. The oil separation efficiency of the fibers was examined by changing the vegetable oil from 2 to $10 \mathrm{v} / \mathrm{v} \%$ in oil-polluted water. All the fibers showed $>98 \%$ of separation efficiency independent of the f-PS surface texture in $2 \%$ oil-polluted water. Increasing the oil concentration to 5 and $10 \%$ reduces this ability to 85 and $82 \%$, respectively for the f-PS fabricated from 1:3 DMF to THF ratio. Thus, the performance of the oil separation is mainly governed by the morphology of the individual fibers and electrospun mats. The fibers prepared from 20 -wt\% concentration in DMF:THF (1:3) mixture offers the best oil sorption, this is the fiber system showing the high adsorption capacity among all samples owing to the having interior porosity and surface texture.

Fig. 8(b-f) shows the photographic and optical microscopy images of oil droplets before and after the treatment with f-PS fibers. The oil droplets were disappeared after the treatment and only little amount of small droplets were remained in the water. These droplets might arise from the remaining volume of the oil droplets after separation. The disappearance of large amount of the droplets may support the hypothesis that the electrospun fibers of f-PS wastes are effective for the removal of oil from water. In order to investigate the practical application of oil separation efficiency of f-PS fibers, we prepared an engine oil/water mixture by using waste engine oil and compared our results with the real crude oil-water mixture obtained from a big Shipyard in Izmir (one of the biggest in Europe). Fig. 9 shows the comparison of oil separation efficiency of pristine foam, commercial adsorbent, and f-PS fibers fabricated from 20-wt\% PS solution in 1:3 (DMF:THF) solvent system. The separation efficiency of adsorbents was investigated for the engine oil/ water mixture $(10 \%, v / v)$. The f-PS fiber mats show $82 \%$ separation efficiency while the pristine foam and commercial adsorbent have 16 and $44 \%$ under same circumstances, respectively. Hereby, the f-PS adsorbents can be a promising candidate for the remediation of oil spills compared to the commercial adsorbent.

The literature also shows the fabrication of electrospun sorbets for oil water separation. In Table 2, the sorption capacity of the electrospun fibers from previously reported literature is given. AFD of the fibrous sorbents reported in the relevant literature and current work is, in general, on the order of several $\mu \mathrm{m}$. The thickness of the fibers is comparable. The f- PS fibers fabricated in this study show higher oil sorption capacity than any type of sorbent materials prepared by electrospinning of polymers without/with functional additives such as magnetic nanoparticles, carbon nanotubes, etc. The reason of the better performance is unclear at the moment because none of the spectroscopic measurements offered a clear difference between the chemical composition of foam-waste and virgin PS (Fig. S4 and S5). Note that the error bars in Fig. 9 are small suggesting that the results are reproducible.

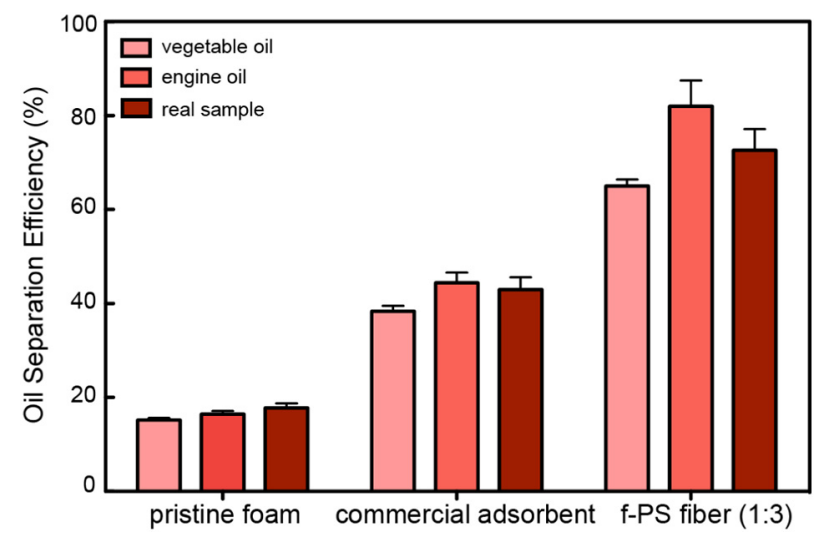

Fig. 9. Oil separation efficiency comparison of pristine foam, commercial adsorbent and proposed f-PS fiber (1:3) by using vegetable oil, engine oil, and real oily wastewater sample. 
Table 2

Comparison of oil adsorption capacities of polymeric electrospun fibers.

\begin{tabular}{|c|c|c|c|c|}
\hline Electrospun material & $\mathrm{AFD}(\mu \mathrm{m})$ & Oil type & Adsorption capacity (g/g) & Ref. \\
\hline Polyvinylidene fluoride & 0.055 & Soybean oil & 14 & [81] \\
\hline Polyvinylidene fluoride / Polystyrene & 2 & Vegetable oil & 46 & [81] \\
\hline Polyvinylidene fluoride / Polystyrene / $\mathrm{Fe}_{3} \mathrm{O}_{4}$ & 2 & Soybean oil & 35 & [81] \\
\hline Polysulfone & 1 & Engine oil & 39 & [82] \\
\hline Polyimide & 3 & Engine oil & 76 & [83] \\
\hline Polystyrene & 3 & Vegetable oil / engine oil & $97 / 114$ & [47] \\
\hline Polystyrene / $\mathrm{Fe}_{3} \mathrm{O}_{4}$ & 1 & Soybean oil & 70 & {$[81]$} \\
\hline Polystyrene from foam waste & 4 & Vegetable oil / engine oil & $124 / 95$ & This study \\
\hline
\end{tabular}

The main difference of the current work from the relevant literature $[45,46,80]$ is the employment of the waste plastics for the preparation of the sorbent materials. The starting material in the literature is, all without exception, virgin polymer. Such being the case, the separation of oil for the environmental concern causes the formation of another environmental issue in long run since the synthetic virgin plastic is employed. The usage of the wastes minimizes the consumption of plastics that absolutely brings an indirect environmental benefit. On the other hand, needless to say that virgin plastics have much higher price than the waste one, this usage prevents a remarkable economical loss. Another advantage of this report from the literature is the rapid sorption process. Sorption time is extremely critical parameter when a ship accident occurs. A competition against time takes place to clean water and prevent invasion of the oil into sea/oceans. The usual sorption time for lab-scale experiments for complete removal of oil from water in literature lies in between 5 and 8 min depending on the type of the contaminant oil, composition of oil-water mixture, and the volume of the mixture treated with fibers $[45,46,80]$. The time for sorption in this study is reduced to $<1 \mathrm{~min}$ in lab-scale trials. This rapid sorption can be explained by the formation of both surface and interior porosity of the individual fibers obtained by electrospinning process from a particular solvent composition of DMF and THF (1:3) along with chemical affinity between PS and oil molecules. Mapping of the fiber morphology against the solvent composition and polymer concentration is provided. The porous structure mediates the sorption process of oil molecules in high amount. This short comparison of oil adsorption capacities through the literature strongly suggests that the f-PS fibers fabricated from waste products can be an efficient and low-cost alternative to the oil separation from water systems.

\section{Conclusion}

EPS wastes were readily processed by electrospinning from the solvent mixture of THF and DMF. Freestanding and highly flexible fiber mats were achieved and used as adsorbent materials for oil water separation. A detailed phase diagram is proposed for the determination of fiber morphology (diameter, shape, and surface and interior porosity) as a function of the co-solvent composition and polymer concentration of the precursor electrospinning solution. While THF-rich solvent mixtures cause the formation of surface porosity due to the breath figure formation, the DMF-rich ones facilitate the formation of pores both on the surface of the fibers and interior pores in their cross section due to the contribution of VIPS and TIPS. This variation of surface morphology remarkably influences the hydrophobicity/oleophilicity of the fiber mats, i.e. sorption capacity against oily wastewaters. The f-PS fibers show better oil adsorption and separation performance than the pristine foam does. The maximum vegetable oil adsorption capacity of f$\mathrm{PS}$ reaches up to $124 \mathrm{mg} / \mathrm{mg}$ and they have nearly 93\% oil separation efficiency for highly oil-polluted mixtures (10\% oil by volume). For low oil concentrations, they could clean nearly the whole vegetable oil from the water with $98 \%$ efficiency. It must also be said that the f-PS mat depicts 4 times higher separation efficiency than the commercial ones when tried in real oily water samples taken from the Izmir Shipyard. Finally, this work may provide a versatile avenue for the fabrication of adsorbents, which are excellently suited for recycling of EPS wastes and efficient separation of various oil polluted waters.

Supplementary data to this article can be found online at https://doi. org/10.1016/j.susmat.2018.e00084.

\section{Acknowledgement}

The authors thank the İzmir Institute of Technology Center for Materials Research and IKCU Central Research Laboratory.

\section{References}

[1] L. Feng, Z.Y. Zhang, Z.H. Mai, Y.M. Ma, B.Q. Liu, L. Jiang, D.B. Zhu, A superhydrophobic and super-oleophilic coating mesh film for the separation of oil and water, Angew. Chem. Int. Ed. 43 (2004) 2012-2014.

[2] J.P. Chaudhary, S.K. Nataraj, A. Gogda, R. Meena, Bio-based superhydrophilic foam membranes for sustainable oil-water separation, Green Chem. 16 (2014) 4552-4558.

[3] B. Wang, W.X. Liang, Z.G. Guo, W.M. Liu, Biomimetic super-lyophobic and superlyophilic materials applied for oil/water separation: a new strategy beyond nature, Chem. Soc. Rev. 44 (2015) 336-361.

[4] S.K. Zhou, P.P. Liu, M. Wang, H. Zhao, J. Yang, F. Xu, Sustainable, reusable, and superhydrophobic aerogels from microfibrillated cellulose for highly effective oil/ water separation, ACS Sustain. Chem. Eng. 4 (2016) 6409-6416.

[5] M.A. Shannon, P.W. Bohn, M. Elimelech, J.G. Georgiadis, B.J. Marinas, A.M. Mayes, Science and technology for water purification in the coming decades, Nature 452 (2008) 301-310.

[6] Oil in the Sea III, Inputs, Fates, and Effects, Transportation Research Board and National Research Council, Washington, D.C 2003.

[7] T. Krebs, C. Schroen, R.M. Boom, Separation kinetics of an oil-in-water emulsion under enhanced gravity, Chem. Eng. Sci. 71 (2012) 118-125.

[8] L. Adam, C. Puscasu, N. Constantin, S. Neacsu, M. Butu, F.G. Dinu, New procedures and methods in chemical treatment and centrifugal separation field in wastes components of oil industry, Rev. Chim. 60 (2009) 1103-1106.

[9] V.K. Rajak, K.K. Relish, S. Kumar, A. Mandal, Mechanism and kinetics of separation of oil from oil-in-water emulsion by air flotation, Pet. Sci. Technol. 33 (2015) 1861-1868.

[10] Y.Y. Zheng, C.C. Zhao, A study of kinetics on induced-air flotation for oil-water separation, Sep. Sci. Technol. 28 (1993) 1233-1240.

[11] R.C. Little, Chemical demulsification of aged crude-oil emulsions, Environ. Sci. Technol. 15 (1981) 1184-1190.

[12] Z.X. Xue, Y.Z. Cao, N. Liu, L. Feng, L. Jiang, Special wettable materials for oil/water separation, J. Mater. Chem. A 2 (2014) 2445-2460.

[13] Y. Li, L.L. He, X.F. Zhang, N. Zhang, D.L. Tian, External-field-induced gradient wetting for controllable liquid transport: from movement on the surface to penetration into the surface, Adv. Mater. 29 (2017).

[14] Z.L. Chu, Y.J. Feng, S. Seeger, Oil/water separation with selective superantiwetting/superwetting surface materials, Angew. Chem. Int. Ed. 54 (2015) 2328-2338.

[15] Z. Tan, Y. Liang, H. Chen, D.J. Wang, Synthesis of hexadecyl methacrylate/methyl methacrylate copolymer by high internal phase emulsion template and its high oil-absorbing properties, Sep. Sci. Technol. 48 (2013) 2338-2344.

[16] K.Q. Li, X.R. Zeng, H.Q. Li, X.J. Lai, Facile fabrication of a robust superhydrophobic/ superoleophilic sponge for selective oil absorption from oily water, RSC Adv. 4 (2014) 23861-23868.

[17] Q.F. Xu, J.N. Wang, K.D. Sanderson, Organic-inorganic composite nanocoatings with superhydrophobicity, good transparency, and thermal stability, ACS Nano 4 (2010) 2201-2209.

[18] W.B. Zhang, F. Zhang, S.J. Gao, Y.Z. Zhu, J.Y. Li, J. Jin, Micro/nano hierarchical poly (acrylic acid)-grafted-poly(vinylidene fluoride) layer coated foam membrane for temperature-controlled separation of heavy oil/water, Sep. Purif. Technol. 156 (2015) 207-214.

[19] U. Manna, D.M. Lynn, Synthetic surfaces with robust and tunable underwater superoleophobicity, Adv. Funct. Mater. 25 (2015) 1672-1681.

[20] Y. Li, L. Li, J.Q. Sun, Bioinspired self-healing superhydrophobic coatings, Angew Chem. Int. Ed. 49 (2010) 6129-6133. 
[21] L. Jiang, Z.G. Tang, R.M. Clinton, V. Breedveld, D.W. Hess, Two-step process to create "Roll-off" superamphiphobic paper surfaces, ACS Appl. Mater. Interfaces 9 (2017) 9195-9203.

[22] J.L. Song, Y. Lu, J. Luo, S. Huang, L. Wang, W.J. Xu, I.P. Parkin, Barrel-shaped oil skimmer designed for collection of oil from spills, Adv. Mater. Interfaces 2 (2015).

[23] Y.Q. Qing, C.N. Yang, Z.Y. Yu, Z.F. Zhang, Q.L. Hu, C.S. Liu, Large-area fabrication of superhydrophobic zinc surface with reversible wettability switching and anticorrosion, J. Electrochem. Soc. 163 (2016) D385-D391.

[24] M. Al-Qadhi, N. Merah, A. Matin, N. Abu-Dheir, M. Khaled, K. Youcef-Toumi, Preparation of superhydrophobic and self-cleaning polysulfone non-wovens by electrospinning: influence of process parameters on morphology and hydrophobicity, J. Polym. Res. 22 (2015).

[25] T. Isik, M.M. Demir, C. Aydogan, M. Ciftci, Y. Yagci, Hydrophobic coatings from photochemically prepared hydrophilic polymethacrylates via electrospraying, J. Polymer Sci. 55 (2017) 1338-1344.

[26] A. Celik, G. Koc, E. Erdogan, T. Shahwan, A. Baba, M.M. Demir, Use of electrospun fiber mats for the remediation of hypersaline geothermal brine, Desalin. Water Treat. 62 (2017) 94-100.

[27] M.M. Demir, I. Yilgor, E. Yilgor, B. Erman, Electrospinning of polyurethane fibers, Polymer 43 (2002) 3303-3309.

[28] N. Horzum, E. Boyaci, A.E. Eroglu, T. Shahwan, M.M. Demir, Sorption efficiency of chitosan nanofibers toward metal ions at low concentrations, Biomacromolecules 11 (2010) 3301-3308.

[29] N. Horzum, M.M. Demir, M. Nairat, T. Shahwan, Chitosan fiber-supported zerovalent iron nanoparticles as a novel sorbent for sequestration of inorganic arsenic, RSC Adv. 3 (2013) 7828-7837.

[30] N. Horzum, T. Shahwan, O. Parlak, M.M. Demir, Synthesis of amidoximated polyacrylonitrile fibers and its application for sorption of aqueous uranyl ions under continuous flow, Chem. Eng. J. 213 (2012) 41-49.

[31] T. Isik, N. Horzum, U.H. Yildiz, B. Liedberg, M.M. Demir, Utilization of electrospun polystyrene membranes as a preliminary step for rapid diagnosis, Macromol Mater. Eng. 301 (2016) 827-835.

[32] B. Satilmis, T. Isık, M.M. Demir, T. Uyar, Amidoxime functionalized polymers of Intrinsic Microporosity (PIM-1) electrospun ultrafine fibers for rapid removal of uranyl ions from water, Appl. Surf. Sci. 467-468 (2019) 648-657.

[33] N. Horzum, M. Mari, M. Wagner, G. Fortunato, A.M. Popa, M.M. Demir, K. Landfester, D. Crespy, R. Munoz-Espi, Controlled surface mineralization of metal oxides on nanofibers, RSC Adv. 5 (2015) 37340-37345.

[34] D. Li, Y.N. Xia, Electrospinning of nanofibers: Reinventing the wheel? Adv. Mater. 16 (2004) 1151-1170.

[35] A. Greiner, J.H. Wendorff, Electrospinning: a fascinating method for the preparation of ultrathin fibres, Angew. Chem. Int. Ed. 46 (2007) 5670-5703.

[36] D.H. Reneker, I. Chun, Nanometre diameter fibres of polymer, produced by electrospinning, Nanotechnology 7 (1996) 216-223.

[37] Z.P. Zhou, X.F. Wu, Electrospinning superhydrophobic-superoleophilic fibrous PVDF membranes for high-efficiency water-oil separation, Mater. Lett. 160 (2015) 423-427.

[38] M.H. Jin, J. Wang, X. Yao, M.Y. Liao, Y. Zhao, L. Jiang, Underwater oil capture by a three-dimensional network architectured organosilane surface, Adv. Mater. 23 (2011) 2861.

[39] J. Wu, N. Wang, Y. Zhao, L. Jiang, Simple synthesis of smart magnetically driven fibrous films for remote controllable oil removal, Nanoscale 7 (2015) 2625-2632.

[40] B. Wang, W.W. Chen, L.T. Zhang, Z.Z. Li, C.T. Liu, J.B. Chen, C.Y. Shen, Hydrophobic polycarbonate monolith with mesoporous nest-like structure: an effective oil sorbent, Mater. Lett. 188 (2017) 201-204.

[41] J.Q. Zhang, Q.Z. Xue, X.L. Pan, Y.K. Jin, W.B. Lu, D.G. Ding, Q.K. Guo, Graphene oxide/ polyacrylonitrile fiber hierarchical-structured membrane for ultra-fast microfiltration of oil-water emulsion, Chem. Eng. J. 307 (2017) 643-649.

[42] J.Q. Zhang, X.L. Pan, Q.Z. Xue, D.L. He, L. Zhu, Q.K. Guo, Antifouling hydrolyzed polyacrylonitrile/graphene oxide membrane with spindle-knotted structure for highly effective separation of oil-water emulsion, J. Membr. Sci. 532 (2017) 38-46.

[43] Q. Zhu, Q.M. Pan, F.T. Liu, Facile removal and collection of oils from water surfaces through superhydrophobic and superoleophilic sponges, J. Phys. Chem. C 115 (2011) 17464-17470.

[44] D.S. Yuan, T. Zhang, Q. Guo, F.X. Qiu, D.Y. Yang, Z.P. Ou, A novel hierarchical hollow SiO2@MnO2 cubes reinforced elastic polyurethane foam for the highly efficient removal of oil from water, Chem. Eng. J. 327 (2017) 539-547.

[45] J. Wu, N. Wang, L. Wang, H. Dong, Y. Zhao, L. Jiang, Electrospun porous structure fibrous film with high oil adsorption capacity, ACS Appl. Mater. Interfaces 4 (2012) 3207-3212.

[46] M.W. Lee, S. An, S.S. Latthe, C. Lee, S. Hong, S.S. Yoon, Electrospun polystyrene nanofiber membrane with superhydrophobicity and superoleophilicity for selective separation of water and low viscous oil, ACS Appl. Mater. Interfaces 5 (2013) 10597-10604

[47] J.Y. Lin, Y.W. Shang, B. Ding, J.M. Yang, J.Y. Yu, S.S. Al-Deyab, Nanoporous polystyrene fibers for oil spill cleanup, Mar. Pollut. Bull. 64 (2012) 347-352.

[48] P.Y. Chen, S.H. Tung, One-step electrospinning to produce nonsolvent-induced macroporous fibers with ultrahigh oil adsorption capability, Macromolecules 50 (2017) 2528-2534

[49] M.M. Demir, Investigation on glassy skin formation of porous polystyrene fibers electrospun from DMF, Express Polym Lett 4 (2010) 2-8.

[50] S. Megelski, J.S. Stephens, D.B. Chase, J.F. Rabolt, Micro- and nanostructured surface morphology on electrospun polymer fibers, Macromolecules 35 (2002) 8456-8466.

[51] J. Arnauts, H. Berghmans, Amorphous thermoreversible gels of solutions of atactic polystyrene, in: W. Burchard, B. Ross-Murphy (Eds.) Physical Networks Polymers and Gels, Elsevier Applied Science, London and New York.
[52] C.L. Casper, J.S. Stephens, N.G. Tassi, D.B. Chase, J.F. Rabolt, Controlling surface morphology of electrospun polystyrene fibers: effect of humidity and molecular weight in the electrospinning process, Macromolecules 37 (2004) 573-578.

[53] J.Y. Lin, B. Ding, J.Y. Yu, Y. Hsieh, Direct fabrication of highly nanoporous polystyrene fibers via electrospinning, ACS Appl. Mater. Interfaces 2 (2010) 521-528.

[54] T. Kongkhlang, M. Kotaki, Y. Kousaka, T. Umemura, D. Nakaya, S. Chirachanchai, Electrospun polyoxymethylene: Spinning conditions and its consequent nanoporous nanofiber, Macromolecules 41 (2008) 4746-4752.

[55] P. Vandewitte, P.J. Dijkstra, J.W.A. Vandenberg, J. Feijen, Phase separation processes in polymer solutions in relation to membrane formation, J. Membr. Sci. 117 (1996) $1-31$.

[56] A. Laxminarayan, K.S. McGuire, S.S. Kim, D.R. Lloyd, Effect of initial composition, phase-separation temperature and polymer crystallization on the formation of microcellular structures via thermally-induced phase-separation, Polymer 35 (1994) 3060-3068.

[57] P. Lu, Y.N. Xia, Maneuvering the internal porosity and surface morphology of electrospun polystyrene yarns by controlling the solvent and relative humidity, Langmuir 29 (2013) 7070-7078.

[58] M. Srinivasarao, D. Collings, A. Philips, S. Patel, Three-dimensionally ordered array of air bubbles in a polymer film, Science 292 (2001) 79-83.

[59] C. Shin, Filtration application from recycled expanded polystyrene, J. Colloid Interface Sci. 302 (2006) 267-271

[60] C. Shin, G.G. Chase, D.H. Reneker, Recycled expanded polystyrene nanofibers applied in filter media, Colloids Surf. 262 (2005) 211-215.

[61] C. Shin, G.G. Chase, Separation of liquid drops from air by glass fiber filters augmented with polystyrene nanofibers, J. Dispers. Sci. Technol. 27 (2006) 5-9.

[62] J.F. Zheng, A.H. He, J.X. Li, J.A. Xu, C.C. Han, Studies on the controlled morphology and wettability of polystyrene surfaces by electrospinning or electrospraying, Polymer 47 (2006) 7095-7102.

[63] T. Isik, M.M. Demir, Protein-rich medical waste treatment via waste electrospinning, Fibers Polymers 19 (2018) 767-774.

[64] C.L. Pai, M.C. Boyce, G.C. Rutledge, Morphology of porous and wrinkled fibers of polystyrene electrospun from dimethylformamide, Macromolecules 42 (2009) 2102-2114.

[65] W.J. Liu, C. Huang, X.Y. Jin, Electrospinning of grooved polystyrene fibers: effect of solvent systems, Nanoscale Res. Lett. 10 (2015).

[66] C. Huang, Y.W. Tang, X. Liu, A. Sutti, Q.F. Ke, X.M. Mo, X.G. Wang, Y. Morsi, T. Lin, Electrospinning of nanofibres with parallel line surface texture for improvement of nerve cell growth, Soft Matter 7 (2011) 10812-10817.

[67] W.J. Liu, C. Huang, X.Y. Jin, Tailoring the grooved texture of electrospun polystyrene nanofibers by controlling the solvent system and relative humidity, Nanoscale Res. Lett. 9 (2014).

[68] D.R. Lide, CRC Handbook of Chemistry and Physics, 85 ed. CRC Press, Boca Raton, FL, 2004.

[69] C. Wang, Y.W. Cheng, C.H. Hsu, H.S. Chien, S.Y. Tsou, How to manipulate the electrospinning jet with controlled properties to obtain uniform fibers with the smallest diameter?-a brief discussion of solution electrospinning process, J. Polym. Res. 18 (2011) 111-123.

[70] T. Uyar, F. Besenbacher, Electrospinning of uniform polystyrene fibers: the effect of solvent conductivity, Polymer 49 (2008) 5336-5343.

[71] A. Greiner, J.H. Wendorff, Electrospinning: a fascinating method for the preparation of ultrathin fibers, Angew. Chem. Int. Ed., 46 5670-5703.

[72] M. Bognitzki, W. Czado, T. Frese, A. Schaper, M. Hellwig, M. Steinhart, A. Greiner, J.H. Wendorff, Nanostructured fibers via electrospinning, Adv. Mater. 13 (2001) 70.

[73] A. Ziabicki, Fundamentals of Fibre Formation: The Science of Fibre Spinning and Drawing, John Wiley and Sons, 1976.

[74] J.F. Gao, W. Li, J.S.P. Wong, M.J. Hu, R.K.Y. Li, Controllable morphology and wettability of polymer microspheres prepared by nonsolvent assisted electrospraying, Polymer 55 (2014) 2913-2920.

[75] E.K. Kim, J.Y. Kim, S.S. Kim, Synthesis of superhydrophobic SiO2 layers via combination of surface roughness and fluorination, J. Solid State Chem. 197 (2013) 23-28.

[76] N.A. Patankar, Hydrophobicity of surfaces with cavities: making hydrophobic substrates from hydrophilic materials? J. Adhes. Sci. Technol. 23 (2009) 413-433.

[77] J.Y. Wu, A.K. An, J.X. Guo, E.J. Lee, M.U. Farid, S. Jeong, CNTs reinforced superhydrophobic-oleophilic electrospun polystyrene oil sorbent for enhanced sorption capacity and reusability, Chem. Eng. J. 314 (2017) 526-536.

[78] G. Deschamps, H. Caruel, M.E. Borredon, C. Bonnin, C. Vignoles, Oil removal from water by selective sorption on hydrophobic cotton fibers. 1. Study of sorption properties and comparison with other cotton fiber-based sorbents, Environ. Sci. Technol. 37 (2003) 1013-1015.

[79] J.F. Gao, X. Song, X.W. Huang, L. Wang, B. Li, H.G. Xue, Facile preparation of polymer microspheres and fibers with a hollow core and porous shell for oil adsorption and oil/water separation, Appl. Surf. Sci. 439 (2018) 394-404.

[80] J.Y. Lin, B. Ding, J.M. Yang, J.Y. Yu, G. Sun, Subtle regulation of the micro- and nanostructures of electrospun polystyrene fibers and their application in oil absorption, Nanoscale (4) (2012) 176-182.

[81] Z. Jiang, L.D. Tijing, A. Amarjargal, C.H. Park, K.J. An, H.K. Shon, C.S. Kim, Removal of oil from water using magnetic bicomponent composite nanofibers fabricated by electrospinning, Composites Part B-Engineering 77 (2015) 311-318.

[82] C. Cojocaru, P.P. Dorneanu, A. Airinei, N. Olaru, P. Samoila, A. Rotaru, Design and evaluation of electrospun polysulfone fibers and polysulfone/NiFe204 nanostructured composite as sorbents for oil spill cleanup, J. Taiwan Inst. Chem. Eng. 70 (2017) 267-281

[83] L.D. Tian, C.Y. Zhang, X.W. He, Y.Q. Guo, M.T. Qiao, J.W. Gu, Q.Y. Zhang, Novel reusable porous polyimide fibers for hot-oil adsorption, J. Hazard. Mater. 340 (2017) 67-76. 